\title{
The Relation between the Theory of Mind and Socio-Emotional Functioning in a Sample of Older
}

Adults

\begin{abstract}
Keywords: Theory of Mind (ToM); Mental state attribution; Aging; Socio emotional functioning

Abstract

We explored the relationship between the "Theory of Mind" (ToM) and socio-emotional functioning among primary aging individuals. Our sample was comprised of 266 older adults ranging from 65 years old to 94 years old. On all participants, we measured ToM and calculated indexes of performance such as causal connection and psychologic al lexic on. In addition, we assessed emotional (depression, a lexithymia, distress, life satisfaction, empathy and hostility) and social variables (perception of the future, loneliness, social avoidance and perception of social support) with a battery of tests. The results of our cognitive screening indic ated that 189 older adults presented nomal or primary aging pattems. The results obtained with the sample of nomal older adults showed that: a) social variables are independent from ToM; and b) emotional functioning predicted the making of causal connection and the use of psychological lexicon among partic ipants who responded to ToM tasks. We disc uss our work within a socio-emotional conceptual framework.
\end{abstract}

The Theory of Mind (ToM, henceforth) is the ability to intuit the mental states of others, such as beliefs, desires, social intentions, and overall thinking [1]. ToM helps people with their "reading" of the thinking of others in a probabilistic way (e.g., I believe my colleague is tired now and he is most likely thinking to call for a meeting another day). ToM, which is developed in late childhood, makes it possible to create attributions that are progressively more complex. First, we appraise another person's mental state (as beliefs or thoughts, i.e. 'What John thinks about our friend Zoe'); later we canassess one person's perception about a second person's mental state (i.e. 'What John thinks about what Meg is thinking about Zoe). These attributions require sustained cognitive effort (for a review, see [2]).

Although it is still not clear whether ToM is a distinct cognitive ability (i.e. [3]), which is most likely innate [4], or the end result of a network of abilities [5,6], by consensus there could be positive effects of ToM in the lifespan $[7,8]$, such as a healthier cognitive functioning. The beneficial effects of ToM are generally true for those older adults who maintain higher levels of crystallized intelligence in their later development $[9,10]$. Our main goal in this study is to expand upon these cognitive findings (also reviewed below). More specifically we examined whether older individuals' emotional and social functioning could relate to ToM in a meaningful way.

In fact, it has been established elsewhere that in older adulthood, a time in which declines in information processing speed and working memory seem inevitable, adults increase their life satisfaction by using socio-emotional functioning as a means to create secure and
Journal of

Neurology and Psychology

\section{Lina Pezzuti ${ }^{1 *}$, Emiddia Longobardi ${ }^{1}$, Serena Rossetti ${ }^{1}$, Elena Bartolini ${ }^{1}$, Giulia Natale ${ }^{1}$, Marta Sappino $^{1}$ and Daniele Artistico ${ }^{2}$}

${ }^{1}$ Department of Clinical Psychology, Sapienza University of Rome, Rome, Italy

${ }^{2}$ Department of Psychology, Baruch College - CUNY, New York, USA

*Address for Correspondence

Lina Pezzuti, Department of Clinical Psychology, Sapienza University of Rome Via dei Marsi 78 - 00185 - Rome, Italy, Tel: 0649917622; Fax: 0649917910; E-mail: lina.pezzuti@uniroma1.it

\section{Submission: 04 August 2015}

Accepted: 15 September 2015

Published: 19 September 2015

Copyright: () 2015 Pezzuti L, et al. This is an open access article distributed under the Creative Commons Attribution License, which permits unrestricted use, distribution, and reproduction in any medium, provided the original work is properly cited.

Reviewed \& Approved by: Dr. George T Grossberg, Professor, Department of Neurology \& Psychiatry, Saint Louis University School of Medicine, USA

stable relationships important to their wellbeing $[11,12]$. Because of the paradox of higher rate of cognitive declines with increased self-reports of life satisfaction by the older adults, gerontologists have started to look at components of aging that could explain this paradox. These components, such as the selection of social mates and the ability to regulate emotions with selected friends are germane to ToM. Work by Carstensen et al. [11], for instance, indicated that the degree to which one is aware of her aging process is pivotal when interacting with others to read whether the interaction is meaningful in both "minds". When there is a "positive read," relations are selected to increase one's emotional wellbeing [13].

\section{ToM and aging}

A pioneering work on the effects of aging on ToM, Happés et al. [9] study used a series of ToM stories tasks (Strange Stories Test) that required participants to make inferences about mental states (double bluffs, mistakes, persuasions, and white lies) of a fictional protagonist of the story, and a series of non-ToM stories (control stories task). Happé's et al. [9] findings indicated that performance on ToM tasks is better among older adults (mean age 73 years old) than in younger adults (mean age 21 years old). Importantly, no age difference was found for the control task, in which the mental states of the characters in the stories were not relevant to the required inferences. Happés study was the first to show that the elderly hold superior social insight about ToM. According to Happè et al. [9], "wisdom and social intelligence increase with age, resulting in superior ToM" [14], while other cognitive functions deteriorate in older age.

Successively, Maylor et al. [1] compared the performance scores on ToM tasks of 25 younger adults (mean age 19 years old) to the 
Citation: Pezzuti L, Longobardi E, Rossetti S, Bartolini E, Natale G, et al. The Relation between the Theory of Mind and Socio-Emotional Functioning in a Sample of Older Adults. J Neurol Psychol. 2015;3(2): 7.

ISSN: 2332-3469

performance on the same ToM tasks of two older adult groups (25 older adults mean age 67 years old, and 25 old-older adults mean age 81 years old). They compared these three groups on their comprehension of ToM story tasks and control story tasks. Some of the ToM stories were interwoven with a memory condition in which participants were required to base their answers on their memory of the story. Conversely, some other ToM stories were presented without the memory load condition (participants could answer the question by referring back to the printed story and pictures). The authors showed that the performance of ToM tasks with a memory load was lower for the two older groups. However, when the memory load was not present, the older adults performed better than the younger adults. These results were also confirmed by a second experiment in which Maylor et al. [1] made use of the materials and scoring techniques implemented by Happè et al. [9]. Then these results were also confirmed by the research of Duval et al. and Brunet et al. $[15,16]$.

Recently, Slessor et al. [14] compared 40 younger adults (mean age 20 years old) and 40 older adults (mean age 67 years old) in three different ToM tasks (verbal stories, video-clip stories, black and white photographs of eye expressions) to gauge several aspects of ToM (visual, verbal, and detailed attention to particulars). Results demonstrated that no adults, younger or older had problems with story tasks that used verbal material. However, older participants performed more poorly than younger participants on the ToM tasks that used video and photographs of eye expressions; this suggested that older adults could have a decline in ToM visual tasks. In part, this decline can be explained by a rising difficulty to inhibit irrelevant information. Others, however, believe that ToM declines from younger to older adulthood, independent of age-related changes to domain-general cognitive functioning $[17,18]$.

In a recent meta-analytic review on ToM in aging, Henry and colleagues examined 23 datasets involving 1462 participants (790 younger and 672 older) [19]. They identified six basic types of ToM tasks (stories, eyes, videos, false belief-video, false beliefother, and faux pas) and they categorized each ToM task according to domain (affective, cognitive, or mixed) and modality (verbal, visual-static, visual-dynamic, verbal and visual static, or verbal and visual-dynamic). The results provided evidence for increased ToM difficulties in late adulthood. Furthermore, deficits were evident in older adult subjects across all task types, domains, and modalities.

\section{Neuropsychology of ToM}

Pardini and Nichelli tested differences in ToM among younger to older adults ranging from 20 to 75 years of age and found that, "decline in ToM capacities becomes statistically relevant after 55 years" (p. 103) [20]. These results confirmed that middle adulthood is a time during which mental state reasoning ability begins to decline. However, Pardini and Nichelli used only one task, the Revised Mind in the Eyes Test that requires participants to indicate emotion (choosing one of four emotion words) that a person is exhibiting, based only on one cropped photograph of that person's eyes [20]. One consideration is that these out of context cropped photographs do not translate well into real life scenarios.

Kemp et al. evaluated ToM in normal aging and in patients with various neurodegenerative pathologies (Alzheimer's disease, mild cognitive impairment, frontal and temporal variants of frontaltemporal lobar degeneration and Parkinson's disease) [21]. Patients with these neurodegenerative disorders showed specific deficit in ToM in relation to damage of specific cerebral regions suggesting a neural network (namely a front-subcortical loop linking the basal ganglia to the regions of the frontal lobes) involved in ToM processes. Sandoz et al. obtained similar results in a population with reduced cognitive abilities due to several types of dementia [22].

To shed some clarity on the mixed results regarding the maintenance or decline of ToM during the aging process, Charlton et al. examined the performance of a group of 106 older individuals from middle age to older adulthood (mean age 69 years old) [23] on ToM tasks (with a subset of the story tasks by Happè [9]). Unlike previous studies, Charlton and colleagues correlated ToM measures with other intelligence tests along with axial scans and quantitative measures of brain functioning [23]. The authors used the ToM tasks and measures of verbal intelligence, executive functions and information processing speed. A measure of brain structure through an MRI was also used. The results showed that the performance of ToM tasks declined with age, and the performance on intelligence tests, executive functioning and the speed of information processing affected participants' performance on ToM tasks. These results also indicated that older adults' crystallized intelligence was a significant predictor of ToM performance. According to Mahy et al. verbal ability is a possible compensatory mechanism for completing ToM tasks [10].

In summary, the extant findings point out that (a) ToM should be studied with tasks that consist of faces, cartoons, stories and videos (complex ToM tasks), (b) ToM typically declines in older ages, and (c) the declines are at least partially mediated. In fact, neuroimaging studies suggest that older adults who perform better or as well as younger adults on ToM tasks may activate compensatory mechanisms by drawing upon regions of the brain also associated with crystallized intelligence, emotional intelligence, and interpersonal insight $[9,10,23]$. We predict that people who have a better socio-emotional functioning could also display higher levels of ToM. We constructed our hypothesis based on the evidence that greater verbal fluency (a strong (shared) component of (by) crystallized intelligence, emotional intelligence and interpersonal insight) predisposes individuals to more stable emotion regulation [24]. By contrast, people who are affected by clinical depression or mood disorders are also less able to read their own mental state and those of others (i.e., [25-27]).

To our knowledge, however, there is no prior research that links the socio-emotional functioning in aging and ToM. Albeit exploratory, this research seeks to fill the gap in the literature, by testing whether socio and emotional functioning would predict ToM proficiency in a sample of older adults. Specifically, our goal is to predict the performance obtained on ToM tasks in conjunction to other emotional (depression, alexithymia, distress, life satisfaction, empathy and hostility) and social measures (perception of the future, loneliness, social avoidance and perception of social support). 


\section{Method}

\section{Participants}

We recruited 266 subjects $^{1}$, between the ages of 65 and 94 $\left(\mathrm{M}_{\text {years }}=78.03 ; \mathrm{SD}_{\text {years }}=7.60\right)$ on a volunteer basis. Participants from central Italy were contacted through local agencies such as community centres for the elderly or through personal contacts. At the outset of the recruitment procedures, the Mini Mental State Examination (MMSE, Folstein, Folstein \& McHugh [28]) was administered to detect the presence of subjects with cognitive impairments. We cognitively screened for participants who had a score of 23 points or lower in the MMSE. After screening, we tested our main hypothesis on 189 individuals who presented no cognitive impairment. The 189 subjects were distributed almost evenly for age and gender: 65-74 years old $(n=69 ; M=32, F=37), 75-84$ years old $(n=74 ; M=33, F=41)$, 85-94 years old ( $n=46 ; M=24 F=22$ ). The educational levels across the groups were distributed as follows: $0-5$ years $(n=69 ; M=26, F=43)$; 6-13 years $(n=76 ; M=40, F=36) ; 14-18$ years $(n=44 ; M=23, F=21)$. No educational differences were found in relation to age $\left(\mathrm{F}_{2,186}\right)=2.27$, $\mathrm{p}=106)$.

\section{Measures}

ToM tasks: The stories $\left(n^{\circ} 3\right)$ administered in the present research are the same ones that Baron-Cohen, Leslie and Frith used in a study with autistic children [29]. Unlike the study of Baron-Cohen, Leslie and Frith, the stories were given in the correct order and the subjects were asked to describe what he/she saw. Each story consisted of four illustrations. The first story was called 'mechanical' because it did not foresee the presence of human characters. The second story was called 'behavioural' because there were few human characters in action, however, it was not required to understand their thoughts. The third story was called 'mentalistic' because it assumes that the subject is able to sense the thoughts of the protagonists in the story. The subjects were asked to tell a story based on each set of illustrations. The stories were recorded and then transcribed. In the present study, we used two scores to analyse the data: 1 ) the comprehension of causal connection and 2) the total number of internal state words used as a measure of psychological lexicon (e.g., to think, to believe, to want). These scores were examined for all three of the stories by two independent coders. The few cases of disagreement were further discussed with a third coder to reach a complete agreement $(100 \%)$ on each measure considered.

Mini Mental State Examination (MMSE): The MMSE is a brief examination consisting of eleven questions intended to evaluate a level of cognitive functioning [28]. The MMSE evaluates six areas of cognitive function: orientation, attention, immediate recall, shortterm recall, language, and the ability to follow simple verbal and written commands. The total raw MMSE's scores were corrected with indexes of Magni' table for age and years of education of subjects [30]. A good reliability on our sample (Cronbach's Alpha $=0.91$ ) has emerged.

${ }^{1}$ Originally 280 participants were recruited for the study. However, and for reasons not known to the researchers, 14 subjects did not provide an answer nor complete more than $20 \%$ of the measures in our procedure. Therefore, the final study sample was comprised of 266 participants.
Self-rating Depression Scale (SDS, 20 items): The SDS of Zung is a self-assessment scale designed to investigate the early onset of depression and its items relate to the clinical criteria used in DSM diagnosis of depression [31]. High scores on this scale are indicators of a possible onset of depression. A good reliability on our sample (Cronbach's Alpha $=0.87$ ) has emerged.

Twenty items Toronto Alexithymia Scale (TAS-20, 20 items): The TAS-20 assesses alexithymia, particularly the difficulty in identifying or describing feelings and a cognitive style that is concrete and externally oriented [32]. Higher scores indicate greater difficulty in expressing emotions. The TAS-20 also measures three dimensions as: 1) difficulty identifying feelings; 2) difficulty describing feelings; and 3) externally oriented thinking. The reliability of the scale was satisfactory tested in our sample (Cronbach's Alpha $=0.89$ ).

Life Satisfaction for Elderly Scale: short form (LSES-S 18 items): This scale investigates the overall life satisfaction of individuals and five sub-dimensions of life satisfaction: mood, the meaning of life, daily activities, self and social contact $[33,34]$. The reliability of the scale was satisfactory tested in our sample (Cronbach's Alpha $=0.91$ ).

Future orientation (6 items): Future orientation scale (RLF $\mathrm{OA}-[35,36])$ investigates the perception of older people about their future and the motivation to invest in projects. The reliability of the scale was satisfactory tested in our sample (Cronbach's Alpha $=0.85$ ).

Social Avoidance and Distress-New (SAD-New: 5 items): The SAD-New is a subscale of the 'Social Anxiety Scale for Aging People' $[37,38]$. In particular, the SAD-New assesses social avoidance and distress to new situations or unfamiliar peers. The reliability of the scale was satisfactory tested in our sample (Cronbach's Alpha $=0.80$ ).

Multidimensional Scale of Perceived Social Support (MSPSS): The MSPSS is a scale consisting of 12 items to assess the support received by figures of reference, in particular, family, friends and significant others $[39,40]$. This scale consists of three subscales (family, friends and significant others), each of which consists of four items with a response mode coded on a seven-point scale from 'completely disagree' to 'completely agree'. The reliability of the scale was satisfactory tested in our sample (Cronbach's Alpha $=0.81$ ).

The Interpersonal Reactivity Index: The Interpersonal Reactivity Index is a questionnaire that is based on a concept dispositional of empathy $[41,42]$. The tool is designed to investigate empathy in some behaviour while also assessing for prospective taking and emotional reactivity. It consists of 28 items on a five-point Likert scale (from 'never' to 'always') and is divided into four subscales: empathic imagination (the propensity to identify with others), prospect taking (the ability to adopt the point of view of others), empathic consideration (the tendency to feel compassion) and personal distress (when the viewer experiences a sense of discomfort and anxiety from viewing the unpleasant experiences of others). The reliability of the scale was satisfactory tested in our sample (Cronbach's Alpha $=0.86$ ).

UCLA loneliness scale (UCLA): The UCLA is a test developed by Russell to assess subjective feeling of loneliness, frequency and quality of social relations [43]. The questions were all worded in a negative or "lonely" direction. Participants indicated how often they felt the described emotion on a four point scale that ranged from "never' 
Citation: Pezzuti L, Longobardi E, Rossetti S, Bartolini E, Natale G, et al. The Relation between the Theory of Mind and Socio-Emotional Functioning in a Sample of Older Adults. J Neurol Psychol. 2015;3(2): 7.

ISSN: 2332-3469

to "often." The reliability of the scale was satisfactory tested in our sample (Cronbach's Alpha $=0.85)$.

Loneliness Scale Weiss: developed by Weiss, investigates loneliness through items that reveal emotional loneliness and social loneliness [44]. The reliability of the scale was satisfactory tested in our sample (Cronbach's Alpha $=0.80)$.

\section{Procedure and statistical analyses}

A battery of tests described above was individually administered to each subject. Testing time lasted about forty-five minutes on average. The tests were administered in different random order for each subject. Hierarchical multiple regressions, were performed to examine the associations between ToM and the emotional and social variables. Two hierarchical multiple regressions were carried out on two dependent variables: causal connection and psychological lexicon about ToM stories. In each hierarchical multiple regression, we entered elders' emotional variables (depression, difficulty identifying feelings, difficulty describing feelings, externally oriented thinking, mood satisfaction, life satisfaction, daily living satisfaction, self satisfaction, social contact satisfaction, future orientation and interpersonal reactivity) in the first step and elders' social variables (loneliness, emotional and social loneliness, perception of social support - relatives, friends and significant others, social avoidance and distress) in the second step. The Statistical Package for the Social Sciences (SPSS 20.0) was used to conduct statistical analyses.

\section{Results}

\section{Clinical and normal cases of cognitive declines: A comparison on ToM}

At the outset of the hypothesis testing, we examined scores obtained by our participants in the MMSE. Our examination revealed that 189 participants scored above the critical cut-off of 23 points, 59 participants had a score equivalent to those of mild cognitive impairment (range 21-23 points), and 18 participants scored below 21 points (range 16-20 points), which typically would indicate moderate cognitive impairment. We then examined the mean differences between clinical and normal cases of cognitive declines on the two ToM tasks: (1) causal connection and (2) psychological lexicon.

(1) Participants who scored above the 23 points cut-off of the MMSE, they were normally or primary aging adults, significantly identified $(\mathrm{F}=12.15 ; \mathrm{p}<0.001$; partial eta-squared $=0.08)$ a higher number of causal connections $(\mathrm{M}=0.93 ; \mathrm{SD}=0.87)$ than participants who had mild cognitive impairment $(\mathrm{M}=0.53 ; \mathrm{SD}=0.71)$ or participants with moderate cognitive impairment $(\mathrm{M}=0.17 ; \mathrm{SD}=0.38)$.

(2) Similarly primary aging adults in our study $(\mathrm{M}=4.93$; $\mathrm{SD}=$ 5.62) produced a significant higher number $(\mathrm{F}=3.31$; $\mathrm{p}<0.05$; partial eta-squared $=0.04)$ of internal state words, (psychological lexicon of ToM) than participants who had mild cognitive impairment $(\mathrm{M}=3.98 ; \mathrm{SD}=4.17)$ or with respect participants who had moderate cognitive impairment $(\mathrm{M}=1.83 ; \mathrm{SD}=3.05)$.

The extant literature has in fact confirmed that people who suffer from cognitive declines do not complete ToM tasks with the same success rate of normal older adults (see [23]); therefore, we tested our main hypothesis on the subsample of primary aging individuals.
This is because we are trying to establish whether there is a relation between socio-emotional functioning and ToM in aging, whereby cognitive declines would alter the expected relation [21].

\section{Hypothesis testing}

As shown in Table 1, regarding the causal connection index of the ToM tasks, the emotional variables entered as first block explained $16 \%$ of the variance but three independent variables contributed significantly: depression, externally oriented thinking and the interpersonal reactivity among older adults. Introducing social variables explained an additional $0.9 \%$ of variation in causal connection $\left(\mathrm{R}^{2}\right.$ change $\left.=0.009\right)$. No social variables contributed significantly to explain the variation in causal connection. Together the two sets of independent variables accounted for $17 \%$ of the variance to detect the causal connection in the stories produced by the older adults in the sample. The results indicate that higher scores in detecting the causal connection were predicted by low scores on depression measures, and by having externally oriented thinking, and high interpersonal reactivity or empathy.

With respect to index of psychological lexicon (Table 2) the emotional variables, entered as the first step, accounted the $11 \%$ of the variance. Adding social variables measured in our sample in the second block, did not improved the prediction. All variables together explained $16 \%$ of the variance of the psychological lexicon measured among older adults with ToM tasks. Three emotional constructs contributed significantly: depression, difficulty identifying feelings and interpersonal reactivity.

In particular, high levels of depression, a difficulty identifying feelings and low interpersonal empathy conduce to a reduced ability to use a psychological lexicon.

\section{Discussion}

Our goal was to verify the relations between ToM as measured by story tasks (tell a story about a character in a story) and emotional and social functioning variables in a sample of primary aging individuals. Although we have found null results regarding the relation between social functioning and ToM, we feel we have accomplished most of our goal. We documented that the presence of depression symptoms measured in our primary aging individuals, influences their ability to understand the cause-effect relationship (the causal connection) and the use of inner state language (psychological lexicon) when responding to ToM tasks. Specifically, people who scored higher on depression scales had greater difficulty comprehending the mental states of the fictional protagonist in the story task.

We believe our findings could be possibly explained by the fact that negative moods, which characterize depression states, clutter the understanding of one's mental functioning as well as the understanding of others' mental states. Measures of alexithymia in our sample (such as the difficulty in identifying feelings) were also negatively correlated with the use of psychological lexicon. Additionally, people who reported difficulty in identifying feelings (high scores on the alexithymia scale) used fewer terms referring to a mental state, than people who scored lower on the alexithymia measures.

Overall the results around lower emotional functioning and ToM 
Citation: Pezzuti L, Longobardi E, Rossetti S, Bartolini E, Natale G, et al. The Relation between the Theory of Mind and Socio-Emotional Functioning in a Sample of Older Adults. J Neurol Psychol. 2015;3(2): 7.

ISSN: 2332-3469

Table 1: Hierarchical regression analyses for predicting causal connection in normally aging older adults.

\begin{tabular}{|c|c|c|c|c|c|}
\hline Predictors & B & SE B & $\beta$ & $\mathbf{R}^{2}$ & $\mathbf{R}^{2}$ change \\
\hline Step 1: Emotional constructs & & & & .163 & $.163^{\star *}$ \\
\hline Depression (SDS) & -.02 & .01 & $-.17^{\star}$ & & \\
\hline Difficulty identifying feelings (TAS-20) & .01 & .02 & .06 & & \\
\hline Difficulty describing feelings (TAS-20) & -.02 & .02 & -.09 & & \\
\hline Externally oriented thinking (TAS-20) & -.06 & .01 & $-.32^{\star \star}$ & & \\
\hline Mood satisfaction (LSES-S) & .02 & .03 & .08 & & \\
\hline Daily living satisfaction (LSES-S) & .01 & .04 & .04 & & \\
\hline Self satisfaction (LSES-S) & .08 & .06 & .14 & & \\
\hline Social contact satisfaction (LSES-S) & -.03 & .04 & -.06 & & \\
\hline Future orientation (FO) & -.02 & .01 & -.14 & & \\
\hline Interpersonal reactivity: empaty (IRI) & .01 & .01 & $.16^{*}$ & & \\
\hline Step 2: Social Constructs & & & & .172 & .009 \\
\hline Social loneliness (WEISS) & -.01 & .15 & -.01 & & \\
\hline Emotional loneliness (WEISS) & .12 & .12 & .09 & & \\
\hline Perception of social support: relatives (MSPSS) & .01 & .02 & -.03 & & \\
\hline Perception of social support: friends (MSPSS) & .01 & .01 & .06 & & \\
\hline Perception of social support: significant others (MSPSS) & .01 & .01 & .05 & & \\
\hline Social avoidance and distress (SAD-New) & .01 & .02 & .03 & & \\
\hline Total $\mathbf{R}^{2}$ & & & & .17 & \\
\hline
\end{tabular}

Note. The tabled values for Beta reflect Bs after step 1. ${ }^{*} p<.05,{ }^{* *} p<.001$.

Table 2: Hierarchical regression analyses for predicting psychological lexicon in normally aging older adults.

\begin{tabular}{|c|c|c|c|c|c|}
\hline Predictors & B & SE B & $\beta$ & $\mathbf{R}^{2}$ & $\mathbf{R}^{2}$ change \\
\hline Step 1: Emotional constructs & & & & $.113^{*}$ & $.113^{*}$ \\
\hline Depression (SDS) & -.14 & .08 & $-.20^{*}$ & & \\
\hline Difficulty identifying feelings (TAS-20) & -.25 & .12 & $-.22^{*}$ & & \\
\hline Difficulty describing feelings (TAS-20) & .14 & .14 & .10 & & \\
\hline Externally oriented thinking (TAS-20) & -.10 & .10 & -.08 & & \\
\hline Mood satisfaction (LSES-S) & .06 & .19 & .03 & & \\
\hline Daily living satisfaction (LSES-S) & -.25 & .29 & -.11 & & \\
\hline Self satisfaction (LSES-S) & .43 & .40 & .11 & & \\
\hline Social contact satisfaction (LSES-S) & -.08 & .30 & -.02 & & \\
\hline Future orientation (FO) & -.13 & .10 & -.12 & & \\
\hline Interpersonal reactivity: empaty (IRI) & .08 & .04 & $.16^{*}$ & & \\
\hline Step 2: Social Constructs & & & & .161 & .047 \\
\hline Loneliness (UCLA) & .17 & .10 & .18 & & \\
\hline Social loneliness (WEISS) & -.97 & 1.02 & -.08 & & \\
\hline Emotional loneliness (WEISS) & .45 & .84 & .05 & & \\
\hline Perception of social support: relatives (MSPSS) & -.17 & .11 & -.13 & & \\
\hline Perception of social support: friends (MSPSS) & -.06 & .10 & -.05 & & \\
\hline Perception of social support: significant others (MSPSS) & .04 & .10 & .03 & & \\
\hline Social avoidance and distress (SAD-New) & -.27 & .16 & -.15 & & \\
\hline Total $\mathbf{R}^{2}$ & & & & .16 & \\
\hline
\end{tabular}

Note. The tabled values for Beta reflect Bs after step 1. ${ }^{*} \mathrm{p}<.05,{ }^{* *} \mathrm{p}<.001$.

seem to be in line with previous research on decoding mental states among individuals affected by mood disorders. People who were pessimistic and gloomy (dysphonic college students) were also less successful in decoding the mental states of others [25,26]. Among clinically depressed subjects, "mentalizing" (i.e. understanding what emotions are felt by oneself or another person) and general cognitive functioning are limited [27]. Our work, however, extends upon these findings by showing that the emotional functioning of subjects not affected by mood disorders or clinical depression could predict performance on ToM tasks in a positive way. This was shown among people using a higher number of psychological lexicon utterances who also had higher scores on interpersonal reactivity. Additionally, people who scored high on emotional empathy and interpersonal reactivity also had a higher rate of causal connection on ToM tasks. 
Citation: Pezzuti L, Longobardi E, Rossetti S, Bartolini E, Natale G, et al. The Relation between the Theory of Mind and Socio-Emotional Functioning in a Sample of Older Adults. J Neurol Psychol. 2015;3(2): 7.

ISSN: 2332-3469

\section{Limitations and Future Directions of Research}

In conclusion, we realize that our research is only a preliminary step; as such, it presents inherent limitations. The first limitation is the null effect of social dimension on ToM. Because older adults are typically less inclined to provide exhaustive information by using selfreports only [45], a more experiential method could detect the effect of their social functioning. Also, the social support variables measured in our study may be interacting implicitly with other socio-emotional selectivity patterns. For instance, if our sample of older adults largely answered that they have "less" people in their social support system, this may numerically generate a constantly low response in the data. However, the low number could also have come from the processing of fewer (but higher quality) relations in later life. In future research, if we were to ask older adults to elaborate about the quality of their perceived social support with an open-ended assessment, we would be able to tap into the underlying processes between social functioning and ToM in a more profound way.

Another limitation is about ToM and socio-emotional functioning among secondary aging individuals in our study. People who suffer cognitive declines are an integral part of the aging population, although the success rate with which secondary aging individuals respond to cognitive tests or training is different or lower than primary aging individuals $[12,46]$. We confirmed group differences between secondary and primary aging individuals on ToM tasks, where primary aging individuals performed better. Yet, we recruited only a small number of secondary aging individuals, in addition to not having complete information about their cognitive declines or clinical interviews about their socio-emotional functioning.

Investigators have already documented that cognitive declines affect the relation between ToM and other psychological variables $[21,23]$. Even if we decided to keep our main hypothesis testing specifically for primary aging individuals, we would still suggest to further exploring the relation between ToM and other socioemotional correlates in secondary aging individuals. This could be achieved by: (a) testing a larger number of subjects who suffer from clinically significant cognitive declines, (b) assessing with more diagnostic tools (than just the MMSE) the rate of people's cognitive deficits to see if they are linked to a form of dementia, and (c) using ecological observations to gauge socio-emotional functioning among clinical cases in their natural dwellings.

A final limitation is that we conducted a correlational study. Although promising, our main results about emotional functioning and ToM should be validated within an experimental design methodology. We would suggest investigating the relation between emotional regulation and its positive effects on ToM with specific training sessions. Training sessions could be executed using a story task as described in our study, followed by a group exercise. To mediate the exercise, a practitioner could help focus the discussion about the participants' responses and the mental states of the characters. For instance, participants may be asked to tell a personal episode that match that of the story presented, and invited to report or describe own feelings and thoughts as they would arise during the exercise (see [47]).

Training could also be aimed at increasing emotional response to daily stressors via verbal regulation. It would be necessary to tailor the intervention so as to improve people's ability to buffer against depressive feelings. However, as we learned from our findings, promotion of empathy and interpersonal reactivity may increase the propensity of older adults in understanding the "mind of the other". Hence our final suggestion is to enable, via single session and group exercises, strategies devoted to take into account the other person's feelings first and then trying to intuit what the other is possibly thinking.

\section{References}

1. Maylor EA, Moulson JM, Muncer A, Taylor LA (2002) Does performances on theory of mind tasks decline in old age? Br J Psychol 93: 465-485.

2. Miller SA (2009) Children's understanding of second-order mental states. Psychol Bull 135: 749-773.

3. Baron-Cohen S, Campbell R, Karmiloff-Smith A, Grant J, Walker J (1995) Are children with autism blind to the mentalistic significance of the eyes? $\mathrm{Br} \mathrm{J}$ Dev Psychol 13: 379-398.

4. Leslie AM, Friedman O, German TP (2004) Core mechanisms in 'theory of mind'. Trends Cogn Sci 8: 528-533.

5. de Villiers JG, Pyers JE (2002) Complements to cognition: A longitudinal study of the relationship between complex syntax and false-belief understanding. Cogn Dev 17: 1037-1060.

6. Lohmann $\mathrm{H}$, Tomasello $\mathrm{M}$ (2003) The role of language in the development of false belief understanding: A training study. Child Dev 74: 1130-1144.

7. Carlson SM, Moses LJ (2001) Individual differences in inhibitory control and children's theory of mind. Child Dev 72: 1032-1053.

8. Carlson SM, Moses LJ, Claxton LJ (2004) Individual differences in executive functioning and theory of mind: An investigation of inhibitory control and planning ability. J Exp Child Psychol 87: 299-319.

9. Happè FG, Winner E, Brownell H (1998) The getting of wisdom: theory of mind in old age. Dev Psychol 34: 358-362.

10. Mahy CE, Vetter N, Kuhn-Popp N, Locher C, Krautschuk S, et al. (2014) The influence of inhibitory processes on affective theory of mind in young and old adults. Neuropsychol Dev Cogn B Aging Neuropsychol Cogn 21: 129-145.

11. Carstensen LL, Turan B, Scheibe S, Ram N, Ersner-Hershfield H, et al. (2011) Emotional experience improves with age: evidence based on over 10 years of experience sampling. Psychol Aging 26: 21-33.

12. Freund A, Baltes $P$ (2002) The adaptiveness of selection, optimization and compensation as strategies of life management: evidence from a preference study on proverbs. J Gerontol B Psychol Sci Soc Sci 57: P426-P434.

13. Carstensen LL, Fung HH, Charles ST (2003) Seocioemotional selectivity theory and the regulation of emotion in the second half of life. Motiv Emot 27 103-123

14. Slessor G, Phillips LH, Bull R (2007) Exploring the specificity of age-related differences in theory of mind tasks. Psychol Aging 22: 639-643.

15. Duval C, Piolino P, Bejanin A, Eustache F, Desgranges B (2010) Age effects on different components of theory of mind. Conscious Cogn 20: 627-642.

16. Brunet E, Sarfati Y, Hardy-Bayle MC, Decety J (2000) A PET investigation of the attribution of intentions with a nonverbal task. Neuroimage 11: 157-166.

17. Bernstein DM, Thornton WL, Sommerville JA (2011) Theory of mind through the ages: older and middle-aged adults exhibit more errors than do younger adults on a continuous false belief task. Exp Aging Res 37: 481-502.

18. Sullivan S, Ruffman T (2004) Social understanding: How does it fare with advancing years? Br J Psychol 95: 1-18.

19. Henry JD, Phillips LH, Ruffman T, Bailey PE (2013) A meta-analytic review of age differences in theory of mind. Psychol Aging 28: 826-839.

20. Pardini M, Nichelli PF (2009) Age-related decline in mentalizing skills across 
Citation: Pezzuti L, Longobardi E, Rossetti S, Bartolini E, Natale G, et al. The Relation between the Theory of Mind and Socio-Emotional Functioning in a Sample of Older Adults. J Neurol Psychol. 2015;3(2): 7.

adult life span. Exp Aging Res 35: 98-106.

21. Kemp J, Despres O, Sellal F, Dufour A (2012) Theory of mind in normal ageing and neurodegenerative phatologies. Ageing Res Rev 11: 199-219.

22. Sandoz M, Demonet JF, Fossard M (2014) Theory of mind and cognitive processes in aging and Alzheimer type dementia: a systematic review. Aging Ment Health 18: 815-827.

23. Charlton RA, Barrick TR, Markus HS, Morris RG (2009) Theory of mind associations with other cognitive functions and brain imaging in normal aging. Psychol Aging 24: 338-348

24. Gyurak A, Goodkind MS, Madan A, Kramer JH, Miller BL, et al. (2009) Do tests of executive functioning predict ability to down-regulate emotions spontaneously and when instructed to suppress? Cogn Affect Behav Neurci 9: 144-152.

25. Lee L, Harkness KL, Sabbagh MA, Jacobson JA (2005) Mental state decoding abilities in clinical depression. J Affect Disorders 86: 247-258.

26. Harkness K, Sabbagh M, Jacobson J, Chowdrey N, Chen T (2005) Enhanced accuracy of mental state decoding in dysphoric college students. Cogn Emot 19: 999-1025.

27. Uekermann J, Channon S, Lehmkamper C, Abdel-Hamid M, Vollmoeller W, et al. (2008) Executive function, mentalizing and humor in major depression. J Int Neuropsychol Soc 14: 55-62.

28. Folstein MF, Folstein SE, McHugh PR (1975) "Mini-mental state". A practical method for grading the cognitive state of patients for the clinician. J Psychiatr Res 12: 189-198.

29. Baron-Cohen S, Leslie AM, Frith U (1986) Mechanical behavioural and Intentional understanding of picture stories in autistic children. $\mathrm{Br} \mathrm{J} \mathrm{Dev}$ Psychol 4: 113-125.

30. Magni E, Binetti G, Bianchetti A, Rozzini R, Trabucchi M (1996) Mini-Mental State Examination: a normative study in Italian elderly population. Eur J Neurol 3: 198-202.

31. Zung WW (1965) A self-rating depression scale. Arch Gen Psychiatry 12: 63-

32. Taylor GJ, Bagby RM, Parker JD (1992) The revised Toronto Alexithymia Scale: some reliability, validity and normative data. Psychother Psychosom 57: $34-41$.

33. Laicardi C, Baldassarri F, Artistico D (2001) Unidimensionality of life satisfaction and its relation to social desirability: a confirmatory study of a short form of the Life Satisfaction Scale. Psychol Rep 88: 253-261.
34. Salamon MJ, Conte VA (1984) Manual for the Salamon-Conte Life Satisfaction in the Elderly Scale (LSES). Psychological Assessment Resources, Odessa, Fla.

35. Edelstein BA, Drozdick LW, Ciliberti CM (1999) Assessment of depression and bereavement in older adults. In P. A. Lichtenberg (Ed.), Handbook of geriatric assessment, New York: Wiley, pp. 11-58.

36. Edelstein BA, Heisel MJ, McKee DR, Martin RR, Koven LP, et al. (2009) Development and Psychometric evaluation of the reasons for living--older adults scale: a suicide risk assessment inventory. Gerontologist 49: 736-745.

37. La Greca AM, Lopez N (1998) Social anxiety among adolescents: linkages with peer relations and friendships. J Abnorm Child Psychol 26: 83-94.

38. Pezzuti L, Longobardi E, Milletti K, Ovidi A (2011) A study about the theory of mind in primary and secondary aging. Life Span and Disability 14: 31-44.

39. Zimet GD, Dahlem NW, Zimet SG, Farley GK (1988) The multidimensional scale of perceived social support. J Pers Assess 52: 30-41.

40. Prezza M, Principato MC (2002) La rete sociale e il sostegno sociale. In M. Prezza, \& M. Santinello (Eds.), Conoscere la comunità, Bologna: II Mulino, pp. 193-233.

41. Davis MH (1980) A multidimensional approach to individual differences in empaty. JSAS Catalog of Selected Documents in Psychology 10: 85.

42. Albiero P, Ingoglia S, Lo Coco A (2006) A contribution to the Italian validation of the Interpersonal Reactivity Index. Testing Psicometria Metodologia 13 107-125.

43. Russell DW (1996) UCLA Loneliness Scale (Version 3): reliability, validity and factor structure. J Pers Assess 66: 20-40.

44. Weiss RS (1974) Loneliness: the experience of emotional and social isolation. Cambridge, MA: MIT Press.

45. Carstensen $L$ (2006) The influence of a sense of time on human development. Science 30: 1913-1915.

46. Lindenberger U, Baltes PB (1997) Intellectual functioning in old and very old age: cross-sectional results from the Berlin Aging Study. Psychol Aging 12 : 410-432.

47. Cavallini E, Bianco F, Bottiroli S, Rosi A, Vecchi T, et al. (2015) Training for generalization in the Theory of Mind: a study with older aldults. Front Psychol 6: 1123 